\title{
Prognostic factors for myasthenic crisis after transsternal thymectomy in patients with myasthenia gravis
}

\author{
Atsushi Watanabe, MD \\ Toshiaki Watanabe, MD \\ Takuro Obama, MD \\ Tohru Mawatari, MD \\ Hisayoshi Ohsawa, MD \\ Yasunori Ichimiya, MD \\ Noriyuki Takahashi, MD \\ Katsuyuki Kusajima, MD \\ Tomio Abe, MD
}

From the Department of Thoracic and Cardiovascular Surgery, Sapporo Medical University School of Medicine, Sapporo, Japan.

Received for publication March 18, 2003; revisions requested July 22, 2003; accepted for publication July 28, 2003.

Address for reprints: Dr A. Watanabe, Department of Thoracic and Cardiovascular Surgery, Sapporo Medical University School of Medicine, Sapporo, South 1, West 16, Chuo-ku, Sapporo 060-8543, Japan (E-mail: atsushiw@sapmed.ac.jp).

J Thorac Cardiovasc Surg 2004;127:868-76 $0022-5223 / \$ 30.00$

Copyright $\odot 2004$ by The American Association for Thoracic Surgery

doi:10.1016/S0022-5223(03)01517-4
Objective: The purpose of this study was to assess which clinical features of patients with myasthenia gravis predict postoperative respiratory problems due to myasthenic crisis after transsternal thymectomy.

Methods: One hundred twenty-two patients who underwent transsternal thymectomy in our institute were analyzed retrospectively. Fourteen of those experienced myasthenic crisis and required prolonged (48 hours or more) postoperative mechanical ventilation. The following factors were evaluated: sex, age, body mass index, grade of symptom, disease interval, existence of thymoma, history of preoperative crisis, doses of anticholinesterase drugs, steroid use, pulmonary function, serum anti-acetylcholine receptor antibody, history of pulmonary disease, presence of other disease, operation time, and blood loss.

Results: Univariate analysis revealed preoperative bulbar symptoms (odds ratio $=$ $14.246, P=.001)$, history of preoperative myasthenic crisis $(7.091, .018)$, and preoperative serum level of anti-acetylcholine receptor antibody $>100 \mathrm{nmol} / \mathrm{L}$ $(4.098, .044)$ were prognostic factors for postoperative myasthenic crisis. On the other hand, multivariate logistic regression analysis revealed preoperative bulbar symptoms $(33.333, .004)$, preoperative serum level of anti-acetylcholine receptor antibody $>100 \mathrm{nmol} / \mathrm{L}(7.874, .020)$, and intraoperative blood loss $>1000 \mathrm{~mL}$ $(18.519, .048)$ were prognostic factors for postoperative myasthenic crisis.

Conclusions: In this study, postoperative myasthenic crisis after transsternal thymectomy in 122 patients with myasthenia gravis was affected by the existence of preoperative bulbar symptoms, history of preoperative myasthenic crisis, preoperative serum level of anti-acetylcholine receptor antibody $>100 \mathrm{nmol} / \mathrm{L}$, and intraoperative blood loss $>1000 \mathrm{~mL}$. Meticulous preoperative and postoperative care should be carried out to prevent postoperative myasthenic crisis in patients with these prognostic factors.

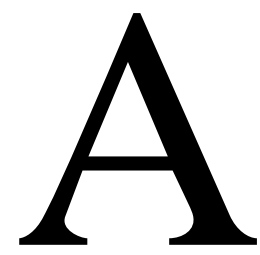

lthough patients with myasthenia gravis may develop respiratory failure for several reasons, it is very difficult to predict myasthenic crisis after transsternal thymectomy. Some scoring systems for predicting myasthenic crisis have been reported by Leventhal and colleagues ${ }^{1}$ and Kimura and coworkers, ${ }^{2}$ and they have been applied in the clinical field. However, the possible influence of serum anti-acetylcholine receptor antibody (AchR-Ab) was not considered in these systems. In our patients, these scoring systems could not always sufficiently predict 
the occurrence of postoperative respiratory failure in patients with myasthenia gravis. There are few studies on the accurate analysis of prognostic factors for postoperative myasthenic crisis, and therefore we sought to determine the effects of clinical features of 122 patients with myasthenia gravis in this study.

\section{Materials and Methods}

\section{Patient Population and Variables Reviewed}

Between January 1985 and December 2002, 122 consecutive patients underwent transsternal thymectomy for myasthenia gravis at our hospital. The patients included 30 men and 92 women and the mean age of $44 \pm 17$ (15-77) years. Their clinical records were reviewed regarding sex, age, body mass index (BMI), grade of symptom (Osserman's classification ${ }^{3}$ ), disease interval, existence of thymoma, history of chronic pulmonary disease (pulmonary emphysema, bronchial asthma, interstitial pneumonia, anthrasilicosis), history of preoperative crisis, preoperative daily dose of anticholinesterase drugs (for convenience, $1 \mathrm{mg}$ of ambenonium chloride or $1 \mathrm{mg}$ of distigmine bromide was considered as $8 \mathrm{mg}$ of pyridostigmine bromide), steroid use, Brinkman index (daily consumption of cigarettes $\times$ years smoked), pulmonary function (vital capacity [VC], \% VC, forced expiratory volume in 1 second [FEV1.0], FEV1.0/forced VC [FEV1.0\%] tidal volume), results of arterial blood gas analysis $\left(\mathrm{PaO}_{2}, \mathrm{PaCO}_{2}\right)$, serum AchR-Ab, resection status of thymoma, operation time, intraoperative blood loss, operative procedure, and postoperative phrenic nerve palsy.

\section{Preoperative Management}

The preoperative regimen of anticholinesterase therapy was maintained until the time of operation. If the patient could not be stabilized with medication, then preoperative plasmapheresis was carried out. Plasmapheresis (blood adsorption) was performed for 7 patients in group I and 3 patients in group II with acute exacerbations and myasthenic crisis. The therapy was used for patients with stable myasthenic symptoms even if their AchR-Ab levels were high because we have no evidence that the therapy reduces the occurrence of postoperative myasthenic crisis, and the cost of these therapies is very high in Japan. Corticosteroids were tapered at remission to the minimal dose required to maintain remission if they were used. The patients with acute exacerbations and myasthenic crisis were not candidates for emergency operations, and thymectomy was avoided until better medical control was gained. Intravenous immune therapy was not carried out in this series.

\section{Anesthetic Management}

A thoracic epidural catheter was inserted in the Th5-6 or Th6-7 (thoracic vertebrae) interspace. Endotracheal intubation was performed without muscle relaxants in all patients. General anesthesia was induced, and this was followed by controlled ventilation with isoflurane or seboflurane. Anesthesia was maintained with $50 \%$ to $60 \%$ nitrous oxide, $40 \%$ to $50 \%$ oxygen, and isoflurane (or sevoflurane) with epidural bupivacaine $(0.25 \%)$.

\section{Surgical Procedure}

The surgical procedure consisted of transsternal thymectomy between 1985 and 1990 and transsternal extended thymectomy ${ }^{4}$ after
1991. Three surgeons performed these operations in our hospital. Dissection was performed bluntly from pericardium and pleura. If necessary, the pleural cavity was opened and excluded. The borders of extended thymectomy were the diaphragm caudally, the thyroid gland orally, and phrenic nerves laterally. One to 3 chest tubes were placed in the thoracic cavity according to status of the opening of mediastinal pleura. Three chest tubes were placed if bilateral mediastinal pleurae were opened.

\section{Anticholinesterase Therapy}

Each patient underwent serial neurologic examination by a referring neurologist in the early postoperative period. Reinstitution of anticholinesterase therapy was based upon the discretion of the neurologist. Although 112 patients were given a lower dose (about one third or half) of anticholinesterase agents postoperatively compared with the preoperative dosage until the third or fourth postoperative day, the other 10 patients were cases of Osserman's classification I who had a short suffering period or received only corticosteroid therapy because of severe side effects to anticholinesterase agents, so they received no anticholinesterase agents preoperatively. Therefore, they were not given anticholinesterase agents until the third or fourth postoperative day, unless their symptoms deteriorated.

\section{Postoperative Management}

Analgesia was initially maintained with epidural bupivacaine $(0.25 \%)$ immediately after the operation. Before the patient recovered from general anesthesia, arterial blood gas analysis was performed while the patient spontaneously breathed oxygen-enriched air (fraction of inspired oxygen, $\mathrm{FiO}_{2}=0.4$ ). Extubation was performed in the operating room or intensive care unit within 24 hours after the operation if possible. The extubation criteria for weaning from mechanical ventilation were (1) clear consciousness, (2) tidal volume of $5 \times$ body weight $(\mathrm{kg}) \mathrm{mL}$ or more, (3) $\mathrm{PaCO}_{2}$ of $50 \mathrm{~mm} \mathrm{Hg}$ or less under the condition of spontaneous ventilation, (4) $\mathrm{PaO}_{2}$ of $90 \mathrm{~mm} \mathrm{Hg}$ or more, and (5) respiratory rate of 30 breaths/minute or less. If the patient failed to meet these criteria, mechanical ventilation was continued in the form of intermittent mandatory ventilation, the rate being subsequently decreased to a tolerable level, and extubation was later performed if the criteria were met. The criteria for reintubation were (1) respiratory rate of 40 breaths/minute or more, (2) $\mathrm{PaCO}_{2}$ of $55 \mathrm{~mm} \mathrm{Hg}$ or more, and (3) tidal volume of $4 \times$ body weight $(\mathrm{kg}) \mathrm{mL}$ or less.

\section{Definition of Postoperative Myasthenic Crisis}

Postoperatively all patients received chest physical therapy, including incentive spirometry, chest clapping and squeezing, intermittent positive-pressure breathing, aerosols, and endotracheal or transnasal suctioning, if necessary. In this study, postoperative myasthenic crisis was defined as total postoperative mechanical ventilation support time of more than 48 hours with no postoperative cardiopulmonary complications or cholinergic crisis. Respiratory failures due to postoperative phrenic nerve palsies were excluded from postoperative crisis. Postoperative phrenic nerve palsies were diagnosed by the finding on chest roentgenogram on fully inspiratory and expiratory phase and/or a phrenic nerve conduction study (the phrenic nerve was cutaneously stimulated by electrical pulse current at the midpoint of the posterior border of 
TABLE 1. Patients with postoperative myasthenic crisis (n = 14)

\begin{tabular}{lcc}
\hline & No. & $\%$ \\
\hline $\begin{array}{l}\text { Failure of extubation }<48 \\
\quad \text { hours }\end{array}$ & 2 & 14.3 \\
Rerequirement of PMV after & 12 & 85.7 \\
$\quad$ weaning from PMV & 3 & \\
Preoperative blood adsorption & 7 & 21.4 \\
Postoperative blood & & 50.0 \\
$\quad$ adsorption & 2 & \\
Postoperative tracheostomy & $5.0 \pm 4.7(1-17)$ & \\
Timing of reintubation (day) & 5 & 35.7 \\
$1-2$ & 4 & 28.6 \\
$3-4$ & 4 & 28.6 \\
$5-10$ & 1 & 7.1 \\
17 & $4.8 \pm 2.3(3-109)$ & \\
Duration of PMV (day) & 5 & 35.7 \\
3 & 3 & 21.4 \\
4 & 2 & 14.3 \\
5 & 4 & 28.6 \\
$>6$ & &
\end{tabular}

$P M V$, Postoperative mechanical ventilation.

the sternomastoid muscle, and the diaphragmatic muscle compound action potentials were recorded between the seventh and eighth intercostal space and xiphoid process). ${ }^{5}$ The latter study was performed only for the patients in group II while they had respiratory failure. Myasthenic disorder was diagnosed by the neurologist using neurophysiological examination and/or provocative diagnostic test using intravenous injection of edrophonium chloride (Tensilon).

\section{Follow-up}

The patients were evaluated 3 and 6 months and 1, 3, 5, and 10 years postoperatively. Their clinical status of myasthenia gravis was assessed as: A, no symptoms without medication; B, increased activity with less medication; $\mathrm{C}$, no clinical change; $\mathrm{D}$, more medication, worse symptoms, or both; and E, death due to myasthenia gravis. The remission rate (RR) and palliation rate (PR) were calculated according to the following formulas: $\mathrm{RR}=$ number of $\mathrm{A} /$ total number and $\mathrm{PR}=$ number of $\mathrm{A}+\mathrm{B} /$ total number. $^{6}$

\section{Statistical Analysis}

Statistical analyses were performed using SPSS 10.0 software (SPSS, Inc, Chicago, Ill). All continuous data are expressed as a mean \pm standard deviation of the mean. Categorical and continuous variables were analyzed by the chi-square test (Fisher exact test) as appropriate and by an unpaired Student $t$ test, respectively. Furthermore, continuous variables were converted into categorical variables using each cutoff level and the ratio of the patients with converted variable was compared in the 2 groups. Preoperative and intraoperative variables were used for the determination of statistically significant prognostic factors for postoperative myasthenic crisis. Clinical factors found to have a $P$ value of less than .20 in the univariate analysis were entered into stepwise multiple logistic regression analysis for the determination of statistically significant, independent prognostic factors for postoperative myasthenic crisis.

\section{Results \\ Groups}

Fourteen of the 122 patients (11.5\%) experienced postoperative myasthenic crisis. Two of those 14 patients were unable to be extubated within 48 hours postoperatively, and the other 12 patients underwent tracheal extubation early but subsequently required tracheal reintubation because of respiratory failure. Postoperative reintubation was performed from postoperative days 1 to 17 (day 5 on average), and mechanical ventilation support time averaged 4.8 days (range 3-109). Three patients and 7 patients underwent blood adsorption preoperatively and postoperatively, respectively. Two patients required tracheostomy postoperatively due to long duration of respiratory failure. Tracheal extubation after postoperative myasthenic crisis was successful in all 14 patients. The patients were divided into 2 groups: group I consisted of 108 patients without postoperative myasthenic crisis and group II consisted of 14 patients with postoperative myasthenic crisis (Table 1).

\section{Preoperative Factors}

There were no significant differences between the 2 groups in terms of age, sex ratio, BMI, and disease interval. The number of patients with Osserman's classifications of I, IIA, IIB, and III or more were $25,50,28$, and 5 cases in group I and $1,1,8$, and 4 in group II, respectively $(P=.003)$. Namely, 33 patients in group I and 12 patients in group II had preoperative bulbar symptoms. Four patients in group I and 3 patients in group II had a history of preoperative myasthenic crisis. The ratio of patients with preoperative bulbar symptoms and the ratio of patients with a history of preoperative crisis were significantly higher in group II than in group I. There were no significant differences between the 2 groups in terms of preoperative daily dose of pyridostigmine (156 vs $158 \mathrm{mg}, P=.9670$ ) and ratio of steroid use $(22 / 86$ vs $4 / 10, P=.4945)$. The value of preoperative AchR-Ab was significantly higher in group II than in group I (35.5 vs $204.3 \mathrm{nmol} / \mathrm{L}, P=.0001)$. In preoperative serum level of AchR-Ab, the cutoff level was made every 10 $\mathrm{nmol} / \mathrm{L}$ from 10 to $100 \mathrm{nmol} / \mathrm{L}$ and the variable was converted into categorical variable using the cutoff level; significant difference was found to be greatest between the 2 groups when the cutoff level reached $100 \mathrm{nmol} / \mathrm{L}$. There were 11 patients with AchR-Ab of 100 or more nmol/L in group I and 6 in group II, and the ratio of such patients was higher in group II than in group I $(P=.0046)$. The ratio of patients with an existence of thymoma and history of chronic pulmonary disease were similar in the 2 groups (Table 2). 
TABLE 2. Clinical data

\begin{tabular}{|c|c|c|c|}
\hline \multirow[b]{2}{*}{ Variable } & \multicolumn{2}{|c|}{ Postoperative crisis } & \multirow{2}{*}{$\begin{array}{c}\boldsymbol{P} \\
\text { value }\end{array}$} \\
\hline & $(-)$ : Group I (n = 108) & $(+)$ : Group II (n = 14) & \\
\hline Sex (men/women) & $26 / 82$ & $4 / 10$ & .7448 \\
\hline Age (y) & $44 \pm 16(15-77)$ & $47 \pm 19(20-77)$ & .561 \\
\hline $\mathrm{BMI}$ & $22.9 \pm 3.9(16.8-37.8)$ & $21.1 \pm 3.0(16.8-26.6)$ & .107 \\
\hline$<20$ & $27(25.0 \%)$ & $5(35.7 \%)$ & .5181 \\
\hline \multicolumn{4}{|l|}{ Osserman's classification } \\
\hline I & 25 & 1 & \\
\hline IIA & 50 & 1 & .0001 \\
\hline IIB & 28 & 8 & \\
\hline III+IV & 5 & 4 & \\
\hline Bulbar symptoms & $33(30.6 \%)$ & $12(85.7 \%)$ & .0001 \\
\hline Disease interval (y) & $3.4 \pm 5.9(0.06-36.9)$ & $1.7 \pm 2.4(0.09-8.21)$ & .311 \\
\hline Thymoma & $24(22.2 \%)$ & $5(35.7 \%)$ & .3173 \\
\hline Pulmonary disease & $4(3.7 \%)$ & $1(7.1 \%)$ & .4622 \\
\hline Preoperative crisis & $4(3.7 \%)$ & $3(21.4 \%)$ & .0324 \\
\hline Preoperative dosage of pyridostigmine (mg) & $156 \pm 148(0-840)$ & $158 \pm 126(0-450)$ & .967 \\
\hline Preoperative steroid use & $22(20.4 \%)$ & $4(28.6 \%)$ & .4945 \\
\hline Brinkman index & $147 \pm 346(0-1980)$ & $157 \pm 218(0-585)$ & .921 \\
\hline VC & $3.03 \pm 0.88(0.87-5.22)$ & $2.92 \pm 0.91(1.52-4.40)$ & .687 \\
\hline$\% \mathrm{VC}$ & $102.8 \pm 20.1(38.4-149.4)$ & $94.8 \pm 10.3(76.0-109.3)$ & .181 \\
\hline FEV1.0 & $2.55 \pm 0.83(0.44-4.99)$ & $2.57 \pm 0.90(0.80-3.89)$ & .929 \\
\hline FEV1.0\% & $86.1 \pm 12.1(50.5-134.7)$ & $83.0 \pm 13.5(56.3-98.2)$ & .419 \\
\hline TV & $0.67 \pm 0.23(0.23-1.36)$ & $0.62 \pm 0.19(0.41-1.08)$ & .471 \\
\hline $\mathrm{PaO}_{2}$ & $89.7 \pm 10.1(62.3-100.0)$ & $87.7 \pm 14.6(55.9-100.0)$ & .600 \\
\hline $\mathrm{PaCO}_{2}$ & $39.7 \pm 3.5(31.7-47.9)$ & $41.5 \pm 4.5(29.8-46.9)$ & .185 \\
\hline AchR-Ab level (nmol/L) & $35.5 \pm 67.2(5.2,0-404)$ & $204.3 \pm 344.1(9.3,0-1100)$ & .0001 \\
\hline$>100$ & $11(10.2 \%)$ & $6(42.9 \%)$ & .0046 \\
\hline Operation time & $174 \pm 55(45-365)$ & $203 \pm 98(110-475)$ & .112 \\
\hline Blood loss & $390 \pm 67(60-3600)$ & $393 \pm 302(150-1100)$ & .979 \\
\hline Resection status of thymoma, incomplete resection & $8(7.4 \%)$ & $2(1.4 \%)$ & .322 \\
\hline Thymectomy/extended thymectomy & $32 / 76$ & $5 / 9$ & .758 \\
\hline Phrenic nerve palsy & $5(4.6 \%)$ & $0(0.0 \%)$ & .5837 \\
\hline
\end{tabular}

BMI, Body mass index; $D I$, disease interval; $V C$, vital capacity; $F E V$, forced expiratory volume; $T V$, tidal volume; $A c h R$ - $A b$, anti-acethylcholine receptor antibody.

\section{Pulmonary Function and Blood Gas Analysis}

Preoperative VC (3.03 vs $2.92 \mathrm{~L}, P=.687$ ), FEV1.0 (2.55 vs $2.57 \mathrm{~L}, P=.929)$, and tidal volume $(0.67$ vs $0.62 \mathrm{~L}, P$ $=.471$ ) were similar in the 2 groups. There were no significant differences between the 2 groups with respect to $\mathrm{PaO}_{2}$ (89.7 vs $87.7 \mathrm{~mm} \mathrm{Hg}, P=.600$ ) and $\mathrm{PaCO}_{2}$ (39.7 vs $41.5 \mathrm{~mm} \mathrm{Hg}, P=.185)$. These continuous variables were converted into categorical variables using each cutoff level and the ratios of these converted variables in the 2 groups were compared. There were no significant differences between the ratios in the 2 groups (Table 2).

\section{Operative Factors}

There were no significant differences between the 2 groups with respect to operation time (174 vs 203 minutes, $P=$ .112) and amount of intraoperative blood loss (390 vs 393 $\mathrm{mL}, P=.979)$. These variables were converted into categorical variable using each cutoff level and the ratios of the patients with these converted variables in the 2 groups were compared. There were no significant differences between the ratios in the 2 groups. An intraoperative blood loss $>$ $1000 \mathrm{~mL}$ occurred in 4 patients in group I and 2 patients in group II. All the patients except 1 had invasive thymoma. Bleeding occurred in the other patient following injury of a left brachiocephalic vein. Resection status of thymoma (complete vs incomplete, $P=.322$ ) and the type of thymectomy (thymectomy vs extended thymectomy, $P=.758$ ) did not influence occurrence of postoperative myasthenic crisis (Table 2).

\section{Postoperative Respiratory Complications}

Postoperative phrenic nerve palsies due to intraoperative phrenic nerve injury were diagnosed in 5 patients in group I but no patients in group II. All patients except 1 had thymomatous myasthenia gravis. Five patients in group I and 1 patient in group II developed pneumonia. One patient in group I died from pneumonia 2 months after thymectomy. One patient in group I had hemothorax requiring reoperation for hemostasis. There were no residual pneumothoraxes or pulmonary embolisms in either group. 
TABLE 3. Remission and palliation rates in patients with myasthenia gravis after thymectomy

\begin{tabular}{|c|c|c|c|c|c|c|}
\hline & \multicolumn{6}{|c|}{ Time after thymectomy } \\
\hline & 3 months & 6 months & 1 year & 3 years & 5 years & 10 years \\
\hline \multicolumn{7}{|l|}{ Number } \\
\hline Group I & 107 & 107 & 99 & 87 & 72 & 48 \\
\hline Group II & 14 & 14 & 13 & 11 & 10 & 5 \\
\hline \multicolumn{7}{|l|}{$\operatorname{RR}(\%)$} \\
\hline Group I & 13.1 & 14.9 & 20.2 & 35.6 & 38.9 & 41.7 \\
\hline Group II & 14.3 & 14.3 & 23.1 & 18.2 & 30.0 & 40.0 \\
\hline$P$ value & $>.999$ & $>.999$ & .728 & .325 & .735 & $>.999$ \\
\hline \multicolumn{7}{|l|}{ PR $(\%)$} \\
\hline Group I & 67.2 & 76.6 & 82.8 & 83.9 & 86.1 & 93.8 \\
\hline Group II & 71.4 & 78.6 & 84.6 & 81.8 & 90.0 & 80.0 \\
\hline$P$ values & $>.999$ & $>.999$ & $>.999$ & $>.999$ & $>.999$ & .335 \\
\hline
\end{tabular}

$R R$, Remission rate; $P R$, palliation rate. There are no significant differences between group I and group II regarding to RR and PR in 3 and 6 months and $1,3,5$, and 10 years after thymectomy.

\section{Follow-up Results}

Follow-up results are summarized in Table 3. There are no significant differences between group I and group II regarding RR and PR after 3 and 6 months and 1, 3, 5, and 10 years after thymectomy.

\section{Univariate Logistic Regression Analysis}

Variables that had $P$ values of less than .05 between the 2 groups were preoperative bulbar symptoms (odds ratio $=$ $14.246, P=.001$ ), history of preoperative myasthenic crisis (7.091, 0.018), and preoperative serum level of AchR-Ab > $100 \mathrm{nmol} / \mathrm{L}(4.098,0.044)$ (Table 4).

\section{Multivariate Logistic Regression Analysis}

Clinical factors found to have a $P$ value of less than .20 in the univariate analysis were entered into stepwise multivariate logistic regression analysis for the determination of statistically significant independent prognostic factors for postoperative myasthenic crisis. These factors were: preoperative bulbar symptoms, history of preoperative myasthenic crisis, preoperative serum level of AchR-Ab $>100 \mathrm{nmol} / \mathrm{L}$, intraoperative blood loss $>1000 \mathrm{~mL}$, age $>70$ years, $\mathrm{FEV}_{1.0}<1.2 \mathrm{~L}$, and existence of thymoma. Multivariate analysis revealed preoperative bulbar symptoms (odds ratio $=33.333, P=.004$ ), preoperative serum level of AchR-Ab $>100 \mathrm{nmol} / \mathrm{L}$ (7.874, 0.020), and intraoperative blood loss $>1000 \mathrm{~mL}(18.519,0.048)$ to be prognostic factors for postoperative myasthenic crisis (Table 5).

\section{Limitations of This Study}

The major limitation of the present study was that all data were analyzed retrospectively, a process that mandates caution in the interpretation of the results. There may be some differences in operative procedure because these serial thymectomies were carried out by 3 surgeons over a long period (17 years). Moreover, the number of patients may not be large enough for evaluation by multivariate logistic regression analysis.

\section{Discussion}

Myasthenia gravis is an autoimmune disease characterized by the production of autoantibodies directed against the acetylcholine receptor of the neuromuscular synapse. Autoantibodies directed against the AchR of the motor end plate can be detected in about $85 \%$ of all affected patients. In the remaining $15 \%$, antibodies with specificities to other muscle targets (ryanodine receptor, titin) are involved, which are not detected with the standard assay. ${ }^{7}$ Four treatment modalities are used: anticholinesterase agents to preserve acetylcholine in the synaptic spaces, surgical thymectomy, immunosuppression, and short-term immunotherapies that include phycoerythrin and intravenous immunoglobulin. Treatments directed toward immune regulation improve functional status and reduce the risk of exacerbation. Some exacerbations, called myasthenic crisis, involve the respiratory muscle and are life-threatening. Myasthenic crisis occurs in $15 \%$ to $20 \%$ of patients with myasthenia gravis and is more frequent in the first 2 years of disease. ${ }^{8}$

The nicotinic AchR is the substrate of the autoimmune response. The AchR is a glycoprotein composed of 5 subunits. ${ }^{9}$ Each molecule consists of 2 alpha subunits, 1 beta unit, 1 delta subunit, and 1 gamma epsilon subunit. Autoantibodies are directed at all 5 units but predominantly at the sequences of the alpha subunit, leading to acceleration of the normal degradation of the receptors. In some patients, antibodies may be directed at a site on the alpha subunit different from the major immunogenic subunit. The exact role of this group of potent antibodies is not known but is thought to contribute to development of myasthenic crisis or rapid worsening of muscle strength. ${ }^{10}$ Certain components 
TABLE 4. Univariate regression analysis

\begin{tabular}{|c|c|c|c|}
\hline Variable & $\begin{array}{l}\text { Odds } \\
\text { ratio }\end{array}$ & $95 \% \mathrm{Cl}$ & $P$ value \\
\hline \multicolumn{4}{|l|}{ Age } \\
\hline$>50$ year & 1.919 & $0.630-5.882$ & .254 \\
\hline$>60$ year & 1.130 & $0.289-4.405$ & .861 \\
\hline$>70$ year & 4.329 & $0.717-26.316$ & .110 \\
\hline Sex, female & 0.793 & $0.229-2.741$ & .714 \\
\hline $\mathrm{BMI}<20$ & 1.667 & $0.512-5.435$ & .396 \\
\hline \multicolumn{4}{|l|}{ DI } \\
\hline$>6$ years & 2.688 & $0.331-21.739$ & .355 \\
\hline$>10$ years & 1478.078 & $0.000-5.505 E+28$ & .808 \\
\hline Preoperative bulbar symptom & 14.246 & $3.015-67.300$ & .001 \\
\hline Brinkman Index $>400$ & 0.681 & $0.128-3.623$ & .653 \\
\hline $\mathrm{VC}<2.9 \mathrm{~L}$ & 0.980 & $0.275-3.522$ & .984 \\
\hline \multicolumn{4}{|l|}{$\%$ VC } \\
\hline$<80 \%$ & 0.720 & $0.141-3.685$ & .694 \\
\hline$<70 \%$ & 0.002 & $0.000-5.153 E+16$ & .783 \\
\hline FEV1.0 $<1.2 \mathrm{~L}$ & 3.500 & $0.566-21.647$ & .178 \\
\hline FEV $1.0 \%<70 \%$ & 1.591 & $0.162-15.579$ & .690 \\
\hline History of plumonay disease $(+)$ & 2.000 & $0.207-19.231$ & .549 \\
\hline History of myasthenic crisis & 7.091 & $1.402-35.859$ & .018 \\
\hline Dosage of prestigmine $>300 \mathrm{mg} / \mathrm{d}$ & 484.173 & $0.0-1.354 \mathrm{E}+22$ & .787 \\
\hline Complete resection & 0.480 & $0.081-2.527$ & .386 \\
\hline Preoperative steroid use & 0.844 & $0.212-3.356$ & .810 \\
\hline Operation time $>180 \mathrm{~min}$ & 0.665 & $0.206-2.141$ & .494 \\
\hline \multicolumn{4}{|l|}{ Blood loss } \\
\hline$>500 \mathrm{~mL}$ & 0.503 & $0.139-1.828$ & .297 \\
\hline$>1000 \mathrm{~mL}$ & 4.049 & $0.663-25.000$ & .130 \\
\hline \multicolumn{4}{|l|}{ AchR-Ab level (nmol/L) } \\
\hline$>0.04$ & 1.538 & $0.318-7.435$ & .592 \\
\hline$>10$ & 1.776 & $0.573-5.525$ & .319 \\
\hline$>50$ & 3.112 & $0.967-10.101$ & .057 \\
\hline$>100$ & 4.098 & $1.042-16.129$ & .044 \\
\hline Thymoma $(+)$ & 2.625 & $0.830-8.303$ & .100 \\
\hline
\end{tabular}

$B M I$, Body mass index; DI, disease interval; VC, vital capacity; FEV, forced expiratory volume; $A c h R$-Ab, anti-acethylcholine receptor antibody.

TABLE 5. Stepwise multivariate logistic regression analysis

\begin{tabular}{lccc}
\hline Variable & Coefficient \pm SE & Odds & CI \\
\hline Preoperative bulbar symptom & $-3.508 \pm 1.207$ & 33.333 & $3.135-333.333$ \\
AchR-Ab level $>100$ (nm/L) & $-2.062 \pm 0.886$ & 7.874 & $1.383-45.455$ \\
Blood loss $>1000 \mathrm{~mL}$ & $-2.917 \pm 1.477$ & 18.519 & $1.022-333.333$
\end{tabular}

$A c h R-A b$, Anti-acethylcholine receptor antibody.

of the complement system are thought to potentiate the degeneration of the acetylcholine receptors. The complements $\mathrm{C} 3, \mathrm{C} 9$, and C5-9 activation neoantigens are capable of membrane lysis and are detected at the end plate in myasthenia gravis. ${ }^{11,12}$ Infections, reduction in medication, menstruation, exposure to toxic substances, emotional factors, surgery, thyroid diseases, reactions to contrast agents used with computed tomography of the chest, and the use of some medications (aminoglycosides, tetracycline, musclerelaxing agents, chloroquine, quinine, lithium, chlorpromazine, diazepam, opioids, barbiturates, corticosteroids, thy- roxin, and others) are implicated in the development of crisis. $^{13,14}$

Despite the well-documented role of AchR-Abs in myasthenia gravis, serum levels of AchR-Ab do not correlate with myasthenic crisis ${ }^{13,14}$ or the severity of disease. ${ }^{15}$ There are few reports ${ }^{16}$ on the relationship between serum levels of AchR-Ab and the development of myasthenic crisis following transsternal thymectomy. The present results show that high serum levels of AchR-Abs were implicated in the development of myasthenic crisis after transsternal thymectomy. Our results do not contradict the results 
in previous reports because our patients who developed myasthenic crisis have immunological disorders, especially regarding the complements following physiological and emotional stress due to transsternal thymectomy. In such cases, high levels of AchR-Abs might be directed at a site on the alpha subunit different from the major immunogenic subunit and cause myasthenic crisis. In fact, 0 to $50 \mathrm{nmol} / \mathrm{L}$ of AchR-Abs affected the occurrence of myasthenic crisis after transsternal thymectomy in our series. The death rate of myasthenic crisis has drastically decreased since the 1950 s, from $80 \%$ to less than $10 \%$, thanks to the use of early mechanical ventilation, admission of the myasthenic patient to an intensive care unit, recognition and treatment of precipitating factors, edrophonium testing, use of anticholinesterase drugs, steroid therapy, immunosuppression, plasmapheresis, ${ }^{17,18}$ and intravenous human gamma globulin. ${ }^{7,19}$

We have sometimes seen patients with myasthenic crisis after transsternal thymectomy for myasthenia gravis. The incidence of myasthenic crisis after transsternal thymectomy varies from $6.0 \%$ to $34 \% .^{20-22}$ The comparison of meager data was very difficult because the criteria applied for the calculation of the incidence rates of respiratory insufficiency were not accurately described in any article except one by Kas and coworkers. ${ }^{22}$ Their study showed that $65(33.6 \%)$ of 193 patients who underwent transsternal simple or extended thymectomy developed respiratory failure (defined the same as in our study). The incidence of postoperative respiratory failure in their series is higher than in ours $(11.5 \%)$.

Many methods for postoperative management of myasthenia gravis patients have been reported over the years. Regarding postoperative mechanical ventilation, early workers advocated tracheostomy in all cases, with careful weaning from ventilation. ${ }^{23,24}$ Kas and colleagues ${ }^{22}$ advocated anticholinesterase medication, which was withdrawn for the 48 hours of obligatory postoperative mechanical ventilation. We think that postoperative routine ventilatory support is never necessary and complete withdrawal of anticholinesterase drugs, if they were used for the patient preoperatively, might induce myasthenic crisis despite preventing cholinergic crisis, because the incidence of postoperative respiratory failure in their series is higher as mentioned above. On the other hand, Gorback and colleagues ${ }^{25}$ advocated early extubation after transsternal thymectomy. They reported that appropriate criteria for weaning from mechanical ventilation, first measured at the end of anesthesia, were $\mathrm{PaO}_{2} \geq 90 \mathrm{~mm} \mathrm{Hg}$ under $\mathrm{FiO}_{2}$ of $0.4, \mathrm{PaCO}_{2} \geq$ $50 \mathrm{~mm} \mathrm{Hg}, \mathrm{pH} \geq 7.30$, and respiratory rate $\geq 30$ breaths/ minute, and appropriate criteria for extubation were meeting the criteria for weaning, vital capacity $\geq 10 \mathrm{~mL} / \mathrm{kg}$, and inspiration pressure better than $-30 \mathrm{~cm} \mathrm{H}_{2} \mathrm{O}$. In addition, they reported in their prospective analysis that if extubation was performed on the basis of the criteria, although $\mathrm{VC}$ at the time of extubation decreased to approximately $50 \%$ of preoperative $\mathrm{VC}$, no patients required reintubation after transsternal thymectomy for myasthenia gravis. These criteria seemed to be useful to reduce inappropriate postoperative pulmonary management, but no effective strategy for treating the patients with myasthenic crisis was shown.

The perioperative use of anticholinesterase drugs remains controversial. Pego-Fernandes and colleagues ${ }^{26}$ advocated 50\% reduction in the administration of anticholinesterase drugs on the first postoperative day. On the other hand, Kas and colleagues ${ }^{22}$ advocated cessation of the drugs for 48 hours after thymectomy. The former emphasis was on reducing the occurrence of myasthenic crisis and the latter on reducing the occurrence of cholinergic crisis. We think that a randomized controlled trial is required to assess the effect of postoperative use of anticholinesterase drugs on respiratory failure. If prognostic factors for postoperative myasthenic crisis can be accurately detected preoperatively, it would greatly contribute to the perioperative management of patients with the factors. If proper preoperative and postoperative management is performed, it might be enable us to reduce occurrence of postoperative myasthenic crisis and reduce cases of unnecessary mechanical ventilation support or inappropriate weaning from mechanical ventilation after transsternal thymectomy.

Gracy and colleagues ${ }^{17}$ reported that the grade of preoperative bulbar symptoms is the only predictive factor for myasthenic crisis after transsternal thymectomy. On the other hand, Loach and colleagues ${ }^{27}$ reported that preoperative vital capacity of less than $2.0 \mathrm{~L}$, presence of thymoma, presence of preoperative bulbar symptoms, and age of more than 50 years influenced myasthenic crisis. Leventhal and colleagues ${ }^{1}$ established a scoring system to predict requirement for postoperative mechanical ventilation after thymectomy by evaluation of 24 patients. In this system, 12 points are assigned to disease interval $>72$ months, 10 points to history of respiratory disease, 8 points to dosage of pyridostigmine $>750 \mathrm{mg} / \mathrm{d}$, and 4 points to $\mathrm{VC}<2.9 \mathrm{~L}$. A total score of 10 or more points predicted the requirement for postoperative mechanical ventilation. Although prognostic factors for postoperative myasthenic crisis have been reported and used in clinical management, ${ }^{1,2}$ the possible influence of serum AchR-Ab was not considered in these reports despite its wide clinical use as a diagnostic parameter of myasthenia gravis.

We evaluated the effects of clinical features of patients with myasthenia gravis and the effect of serum AchR-Ab on postoperative myasthenic crisis. Univariate analysis in the present study indicated that 3 preoperative factors influenced myasthenic crisis after transsternal thymectomy in patients with myasthenia gravis: (1) existence of preoperative bulbar symptoms, (2) history of preoperative myasthenic crisis, and (3) preoperative serum AchR-Ab $>100$ 
$\mathrm{nmol} / \mathrm{L}$. On the other hand, multivariate logistic regression analysis revealed existence of preoperative bulbar symptoms, preoperative serum level of AchR-Ab $>100 \mathrm{nmol} / \mathrm{L}$, and intraoperative blood loss $>1000 \mathrm{~mL}$ were prognostic factors for postoperative myasthenic crisis. Although it is not clear how intraoperative blood loss $>1000 \mathrm{~mL}$ affects postoperative myasthenic crisis, stress and immune disorder caused by a large amount of blood loss might be associated.

Although the risk of respiratory failure may have been decreased by using less invasive surgical approaches (cervicotomy, ${ }^{28-30}$ partial sternotomy, ${ }^{26}$ or video-assisted thoracoscopic surgery ${ }^{31,32}$, avoiding myorelaxants and longacting anesthetic agents, and providing adequate postoperative analgesia, ${ }^{33,34}$ the accurate incidence rate of postoperative myasthenic crisis was not described in these reports. Contraindications to cervical thymectomy include advanced age, poorly controlled neurologic symptoms, and evidence for a thymoma. The procedure is associated with negligible morbidity and requires only a brief hospitalization. ${ }^{35}$ We must give careful consideration to the point that these procedures remain controversial as to the response rate.

A report by Papatestas and colleagues ${ }^{28}$ on 788 nonthymomatous patients who underwent transcervical thymectomy revealed that the remission rate at 5 years was $23 \%$. Pego-Fernandes and colleagues ${ }^{26}$ reported that thymectomy by partial sternotomy for the treatment of myasthenia gravis revealed that the complete remission rate and improvement rate were $12.7 \%$ and $79.9 \%$, respectively. (They did not describe at what time after thymectomy they calculated these rates.) These results are inferior to ours. On the other hand, Meyers and colleagues ${ }^{29}$ and Shrager and colleagues ${ }^{30}$ recently demonstrated that response rates after transcervical thymectomy were equivalent to the transsternal approach (remission rate at 5 years after the thymectomy was $43 \%$ and $44 \%$, respectively) with significantly less morbidity and only a rare instance of postoperative myasthenic crisis. In all cases, it is important to decrease postoperative mortality and morbidity and to increase response rate after thymectomy.

Gracy and colleagues ${ }^{17}$ and Mahalati and colleagues ${ }^{18}$ revealed that plasmapheresis effectively improved myasthenic crisis. We attempted preoperative plasma adsorption in 2 patients who recently had extended thymectomy for myasthenia gravis and who had 2 or more of the prognostic factors for postoperative myasthenic crisis. Although their myasthenic symptoms improved after the adsorption therapy and preoperative serum level of AchR-Ab reduced to a level below $100 \mathrm{nmol} / \mathrm{L}, 1$ of them required prolonged postoperative mechanical ventilation after transsternal thymectomy due to myasthenic crisis. This result indicates that single plasma adsorption therapy is not always effective in preventing postoperative myasthenic crisis.
Recently, the efficacies of new immunomodulatory therapies, such as double filtration plasmapheresis and intravenous immunoglobulin maintenance therapy, for patients with myasthenia gravis have been reported. ${ }^{36,37}$ The prophylactic efficacy of these therapies on postoperative myasthenic crisis still remains controversial. Establishment of effective preoperative and postoperative management is needed to reduce or eliminate postoperative myasthenic crisis.

In conclusion, existence of preoperative bulbar symptoms, history of preoperative crisis, serum AchR-Ab $>100$ $\mathrm{nmol} / \mathrm{mL}$, and intraoperative blood loss $>1000 \mathrm{~mL}$ influence occurrence of myasthenic crisis after transsternal thymectomy. In patients with these prognostic factors, careful preoperative and postoperative management should be carried out to prevent postoperative myasthenic crisis. Further study is needed to establish the effective preoperative and postoperative management for patients undergoing transsternal thymectomy for myasthenia gravis.

\section{References}

1. Leventhal SR, Orkin FK, Hirsh RA. Prediction of the need for postoperative mechanical ventilation in myasthenia gravis. Anesthesiology. 1980;53:26-30.

2. Kimura M, Kato H, Yuzuhira H, Kiyono S. Scoring system for the prediction of the need for postoperative mechanical ventilation in myasthenia gravis. Jpn J Intensive Care Med. 1985;9:235-41.

3. Osserman KE. Myasthenia gravis. New York: Grune \& Stratton; 1958.

4. Masaoka A, Nagaoka Y, Kotake Y. Distribution of thymic tissue at the anterior mediastinum. Current procedures in thymectomy. J Thorac Cardiovasc Surg. 1975;70:747-54.

5. Lu Z, Tang X, Huang X. Phrenic nerve conduction and diaphragmatic motor evoked potentials: evaluation of respiratory dysfunction. Chin Med J (Engl). 1998;111:496-9.

6. Masaoka A, Yamakawa Y, Niwa H, et al. Extended thymectomy for myasthenia gravis patients: a 20-year review. Ann Thorac Surg. 1996; 62:853-9.

7. Romi F, Skeie GO, Aarli JA, Gilhus NE. Muscle autoantibodies in subgroups of myasthenia gravis patients. J Neurol. 2000;247:369-75.

8. Thomas CE, Mayer SA, Gungor Y, et al. Myasthenic crisis: clinical features, mortality, complications, and risk factors for prolonged intubation. Neurology. 1997;48:1253-60.

9. Changeux JP, Devillers-Thiery A, Chemouilli P. Acetylcholine receptor: an allosteric protein. Science. 1984;225:1335-45.

10. Lewis RA, Selwa JF, Lisak RP. Myasthenia gravis: immunological mechanisms and immunotherapy. Ann Neurol. 1995;37:S51-62.

11. Engel AG, Lambert EH, Howard FM. Immune complexes (IgG and C3) at the motor end plate in myasthenia gravis: ultrastructural and light microscopic localization and electrophysiologic correlations. Mayo Clin Proc. 1977;52:267-80.

12. Engel AG, Arahata K. The membrane attack complex of complement at the end plate in myasthenia gravis. Ann N Y Acad Sci. 1987;505: 326-32.

13. O'Riordan JI, Miller DH, Mottershead JP, Hirsch NP, Howard RS. The management and outcome of patients with myasthenia gravis treated acutely in a neurological intensive care unit. Eur J Neurol. 1998;5:137-42.

14. Younger DS, Raksadawan N. Medical therapies in myasthenia gravis. Chest Surg Clin N Am. 2001;11:329-36.

15. Qureshi AI, Suri MF. Plasma exchange for treatment of myasthenia gravis: pathophysiologic basis and clinical experience. Ther Apher. 2000;4:280-6. 
16. Kodama H, Yoshida I, Ohtani Y, et al. Myasthenia gravis: an analysis of patients with postoperative crisis after thymectomy. Kyobu Geka. 1995;48:110-2.

17. Gracey DR, Divertie MB, Howard FM Jr, Payne S. Postoperative respiratory care after transsternal thymectomy in myasthenia gravis. A 3-year experience in 53 patients. Chest. 1984;6:67-71.

18. Mahalati K, Dawson RB, Collins JO, Mayer RF. Predictable recovery from myasthenia gravis crisis with plasma exchange: thirty-six cases and review of current management. J Clin Apheresis. 1999;14:1-8.

19. Berrouschot J, Baumann I, Kalischewski P, Strker M, Schneider D. Therapy of myasthenic crisis. Crit Care Med. 1997;25:1228-35.

20. Bulkley GB, Bass KN, Stephenson GR, et al. Extended cervicomediastinal thymectomy in the integrated management of myasthenia gravis. Ann Surg. 1997;226:324-35.

21. Hatton PD, Diehl JT, Daly BD, et al. Transsternal radical thymectomy for myasthenia gravis: a 15-year review. Ann Thorac Surg. 1989;47: 838-40.

22. Kas J, Kiss D, Simon V, Svastics E, Major L, Szobor A. Decade-long experience with surgical therapy of myasthenia gravis: early complications of 324 transsternal thymectomies. Ann Thorac Surg. 2001;72: 1691-7.

23. Kirschner PA, Osserman KE, Kark AE. Studies in myasthenia gravis. JAMA. 1969;209:906-10.

24. Osserman KE, Genkins GC. Studies in myasthenia gravis. A review of twenty years' experience in over 1200 patients. Mt Sinai J Med. 1971;38:497-537.

25. Gorback MS, Moon RE, Massey JM. Extubation after transsternal thymectomy for myasthenia gravis: a prospective analysis. South Med J. 1991;84:701-6.

26. Pego-Fernandes PM, de Campos JR, Jatene FB, Marchiori P, Suso FV, de Oliveira SA. Thymectomy by partial sternotomy for the treatment of myasthenia gravis. Ann Thorac Surg. 2002;74:204-8.

27. Loach AB, Young AC, Spalding JMK, Crampton SA. Postoperative management after thymectomy. Br Med J. 1975;1:309-12.

28. Papatestas AE, Genkins G, Kornfeld P, et al. Effects of thymectomy in myasthenia gravis. Ann Surg. 1987;206:79-88.

29. Meyers BF, Cooper JD. Transcervical thymectomy for myasthenia gravis. Chest Surg Clin N Am. 2001;11:363-8.

30. Shrager JB, Deeb ME, Mick R, et al. Transcervical thymectomy for myasthenia gravis achieves results comparable to thymectomy by sternotomy. Ann Thorac Surg. 2002;74:320-7.

31. Yim AP, Kay RL, Izzat MB, Ng SK. Video-assisted thoracoscopic thymectomy for myasthenia gravis. Semin Thorac Cardiovasc Surg. 1999;11:65-73.

32. Mack MJ. Video-assisted thoracoscopy thymectomy for myasthenia gravis. Chest Surg Clin N Am. 2001;11:389-405.

33. Eisenkraft JB, Papatestas AE, Kahn CH, Mora CT, Fagerstrom R, Genkins G. Predicting the need for postoperative mechanical ventilation in myasthenia gravis. Anesthesiology. 1986;65:79-82.

34. Machens A, Busch C, Emskötter T, Izbicki JR. Morbidity after transsternal thymectomy for myasthenia gravis: a changing perspective? Thorac Cardiovasc Surg. 1998;46:37-40.

35. Ferguson MK. Transcervical thymectomy. Chest Surg Clin N Am. 1996;6:105-15.

36. Hilkevich O, Drory VE, Chapman J, Korezyn AD. The use of intravenous immunoglobulin as maintenance therapy in myasthenia gravis. Clin Neuropharmacol. 2001;24:173-6.

37. Yeh JH, Chen WH, Chiu HC. Double filtration plasmapheresis in the treatment of myasthenic crisis-analysis of prognostic. Acta Neurol Scand. 2001;104:78-82. 